\title{
The Acceleration of the Moon and the Universe - the Mass of the Graviton
}

\author{
Göran Henriksson ${ }^{1^{*}}$ \\ ${ }^{1}$ Department of Physics and Astronomy, Uppsala University, Box 516, SE-751 20 Uppsala, Sweden \\ Email: goran.henriksson@physics.uu.se
}

\begin{abstract}
To get accurate knowledge about the longitudinal motion of the Moon we must use the oldest preserved interpretable depictions and texts from the ancient cultures. 33 ancient total or almost total solar eclipses, back to $3653 \mathrm{BC}$, have been used to determine the lunar sidereal secular acceleration in longitude, -30.128 \pm 0.0035 arcseconds/(century) $)^{2}\left(" / \mathrm{cy}^{2}\right)$. The lunar secular acceleration, from the Lunar Laser Range (LLR) measurements, -25.856 $\pm 0.003 / \mathrm{cy}^{2}$, must be corrected for the relativistic effect, -3.604 "/ $\mathrm{cy}^{2}$, in the Earth-Moon inertial system, to get the lunar sidereal secular acceleration, $-29.460 / \mathrm{cy}^{2}$, corrected for General Relativity. The difference between this value and the new calibration, $-0.668 \pm 0.0046 / \mathrm{cy}^{2}$, may be interpreted as the cosmological acceleration by Dvali et al. in a Modified Theory of gravity. This value is equivalent to a Mass of the Graviton $=1.306 \pm 0.009 \times 10^{-56}$ grams and there is no need for the hypothetical Dark Energy.
\end{abstract}

Keywords: Cylinder seal, total solar eclipse, lunar secular acceleration, general relativity, precession of the geodesic, graviton mass.

\section{Introduction}

In section $2-8$, the author presents an analysis of the oldest available observations of total solar eclipses depicted on four Sumerian cylinder seals. The oldest identified total solar eclipse in this investigation took place in $3653 \mathrm{BC}$ and was depicted on a well-preserved protoliterate (4000-3200 BC) cylinder seal, from Eshnunna in the Diyala region in Eastern Iraq, published by Henning von der Osten [1].

My preliminary identification of this total solar eclipse was presented to the post-graduate seminar at the Department of Classical Archaeology and Ancient History at Uppsala University in the spring of 1986. However, at that time the finding place in Iraq of the cylinder seal was not known to me because I had only seen it in a popular science book without references, and it was not obvious at that time that my new computer program for calculation of ancient solar eclipses was good enough. Therefore, it was too early to try to publish the result in a scientific journal. The computer program has now been successfully tested during more than 30 years against all available well-documented total solar eclipses and the new calibration in this paper is independently verified by calculations of total solar eclipses on three other Sumerian cylinder seals.

The Gregorian calendar is used in this paper.

In sections 9 - 14, the author used a 5650-year long time interval with 33 ancient total or almost total solar eclipses to make the first determination of the observed constant value for the lunar sidereal secular acceleration in longitude, without any fitting parameters. This determination has been combined with the LLR-measurements of the lunar radial acceleration to study deviations from the Third Law of Kepler.

It has now been possible to verify the precession of the geodesic in the Earth-Moon inertial system, predicted in Einstein's Theory of General Relativity, and the cosmological acceleration of the EarthMoon system predicted by Dvali et al. in a Modified Theory of gravity, and to determine the Mass of the Graviton, $1.306 \pm 0.009 \times 10^{-56}$ grams.

\section{Cylinder Seal No. 47 in Newell's Collection}


Von der Osten [1] describes the motif on seal No. 47 in the following way: "A bearded god or hero who wears a long garment made of goat- or sheepskin and has two horns protruding from his forehead with a branch or plant between them is seated on a boat, the prow of which consists of the upper portion of a similar figure. Both seem to be rowing. Above them appears an eagle with outspread wings (Imdugud, see below) between two crouching antelopes which it is supposedly holding. Before the boat appears a scorpion-man with a crescent-shaped design above its tail and two globes between tail and body. Behind the boat walks a lion with perhaps a bearded human head; between its hind legs is a globe. Above it appear a pitcher on its side, a plow, and the fore part of a lion devouring an antelope." See Figure 1.

\section{Total Solar Eclipse in Eshnunna 3653 BC}

The main motif on von der Osten's cylinder seal No. 47 (4000-3200 BC), Figure 1, is, according to the present author, a depiction of the sky visible during a total solar eclipse after sunrise on 12 September, in $3653 \mathrm{BC}$, at 06.34 local mean solar time in Eshnunna, the main ancient city in the Diyala region, Figure 2 .

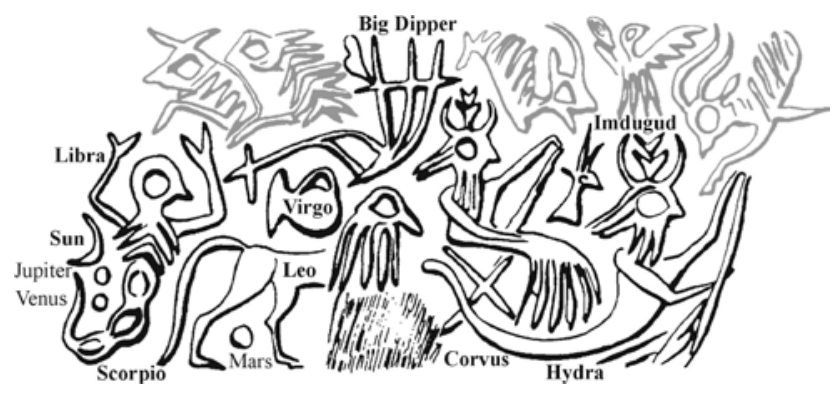

Figure 1. A protoliterate (4000-3200 BC) cylinder seal from the Diyala region, north-east of Baghdad. Drawing after seal No. 47 in von der Osten [1]. Identifications by the author. Marble, $29 \times 17 \mathrm{~mm}$.

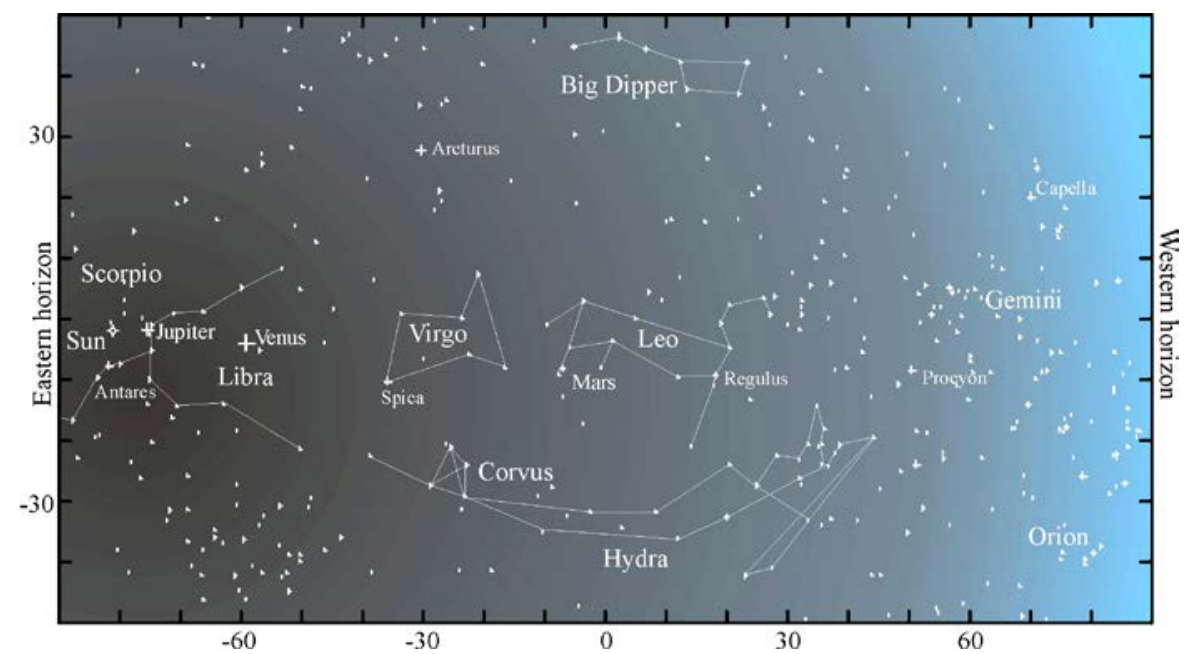

Figure 2. The sky during the total solar eclipse in Eshnunna, on 12 September, in 3653 BC, at 06.34 local mean solar time. Jupiter was visible in Scorpio and Venus in Libra close to the sun. Mars was visible between the hind legs of Leo. The lines between the stars are an attempt to reconstruct the old Sumerian constellations on the protoliterate cylinder seal No. 47 (von der Osten [1]). (Zenith projection, stellar magnitudes < 5.0.) The artist has tried to compress the constellations along the ecliptic visible during the total phase of the solar eclipse. The lower right half of the picture shows the sun-god in his boat that consists of our constellation Hydra, and the throne in the back corresponds to Corvus. 
The lion (Leo) is standing behind the boat, perhaps because there is no space at its normal position on the sky, Figures 2 and 3. On the other hand, in this way all the ecliptic constellations, visible during the eclipse, have been depicted together. On a later seal, Figure 4, the lion is placed in the boat and tied to the prow. The pitcher above the lion, our Virgo, should have been depicted behind the lion, but it was more important to get the space needed for the Scorpion-man, our Libra and Scorpio, where the sun and the planets Jupiter and Venus were visible above each other during the total phase of the eclipse, Figure 2 .

The fact that the much fainter planet Mars has been correctly depicted between the hind legs of Leo is a strong independent verification of the correctness of the interpretation.

The plough, may be the Big Dipper, visible in the north during the totality. It has its normal position above Leo, as in the sky, but there exists also a later Babylonian constellation MUL.APIN, meaning plow.

The eagle in the upper right corner with a goat on each side can be identified as Imdugud (Anzu). Henry Frankfort [2], has identified Imdugud on many cylinder seals. This demon, half man and half bird, stole the "Tablets of Destiny" from Enlil. Anu ordered the other gods to retrieve the tablets, even though they all feared the demon.

\section{Sun-God in His Boat}

Frankfort [2] gives the following description of Sun-god in his boat on page 33: "Even if the same subject is treated, the manner of its rendering is vastly different. The designs on Nos. 331 (ED IIIa) and 621 (Akk.) represent the god in his boat; the designs differ only in the subsidiary motifs added to the main elements. The god (definitely characterized as the sun-god on the Akkadian seal) holds the steering oar of the boat with its human-shaped prow."

The sun-god in his boat on seal No. 47 is depicted when the sun was situated in Leo at the summer solstice, around $3500 \mathrm{BC}$. This is probably the original epoch for this motif, preserved on the cylinder seals during more than 1000 years, Figure 4.

Agricultural attributes are depicted above the back of the lion: the pitcher with handle, the plow, on No. 621 perhaps a bag of seed, and behind the boat a goddess of vegetation, characterized by ears of grain growing from shoulder and robe. This part of the ecliptic corresponds to our constellation Virgo holding an ear in her hand. Spica, the brightest star in Virgo, means ear in Latin.

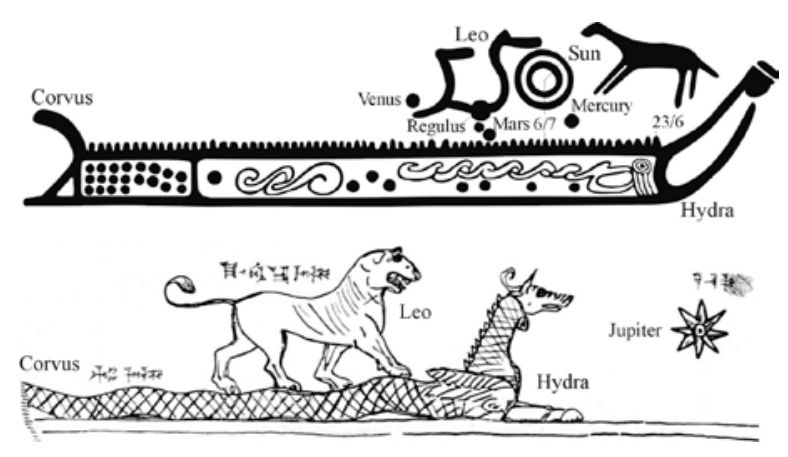

Figure 3. The upper figure is a Swedish rock-carving from the Bronze-Age, at Ekenberg, in Norrköping. It depicts the total solar eclipse on 6 July, 1230 BC, with the sun in front of Leo and the planets as cup marks, Henriksson [3], [4]. The boat from Ekenberg corresponds to the boat of the sun-god on the cylinder seals. The seat of the sun-god is Corvus and the prow is the head of Hydra.

The lower figure can be found in a cuneiform text from the Arsacid dynasty (247 BC - AD 224), an ancient Iranian dynasty that ruled the Parthian empire. VAT 7847 in Berlin. This is a mirror image to get the same orientation as in the sky. (Jeremias [4]). 


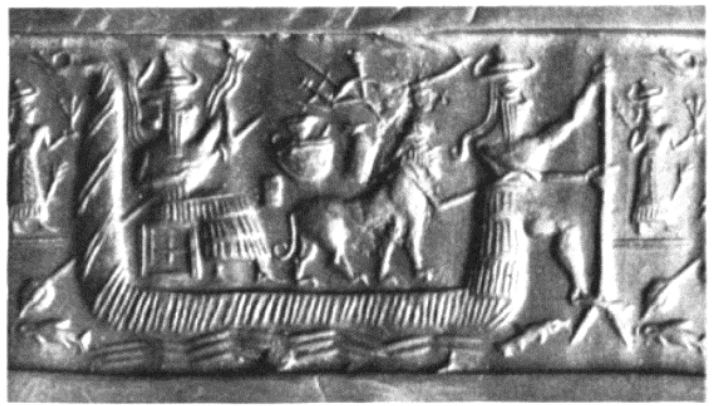

Figure 4. Frankfort seal No 621: "Sun-god in his boat holds steering oar; anthropomorphous prow, with longhaired crowned figure using punting pole and snake-head stern; human-headed lion in boat, tied to prow; above lion, plow, vase with spout and handle, and two unrecognizable objects, one of which is perhaps a bag of seed; outside boat, goddess of vegetation, characterized by ears of grain growing from shoulder and robe, holds flowering branch; fishes and zigzags meaning water below." Frankfort [2]. Akkadian style, 2350-2150 BC. Shell; 3.7 x $2.1 \mathrm{~cm}$.

\section{$5 \quad$ Identified Astronomical Symbols on the Cylinder Seals}

Von der Osten [1] has identified many objects on the cylinder seals. A disk with four internal rays and a crescent at the lower limb or just a crescent is always a solar symbol. But the sun is only visible as a crescent during solar eclipses which means that these objects are in fact symbols for solar eclipses. A close group with seven drill-holes is the Pleiades. Individual drill-holes may be planets. A star like object may be a bright planet Venus. A disk with star like rays may be the head of a bright comet.

\section{Total Solar Eclipse in 2654 BC}

The main motif on cylinder seal No. 331 is "Sun-god in his boat", Frankfort [2]. This seal is from ED IIIa, 2600-2500 BC, Figure 5.

I identify the motif on Frankfort No. 331, as a depiction of the sky visible during the total solar eclipse in 2654 BC, just outside the archaeological date 2600-2500 BC for ED IIIa, Figure 5, 6.

The crescent is the sun, the star with disk is Comet Encke and the drill-holes closest to the sun are Mercury, Mars and Venus.

The unique position of Comet Encke, below the sun, depicted as a star with a disk and without tail, confirms the identification. The tail was hidden behind the nucleus and coma because the comet was that day moving towards the Earth along the line of sight.

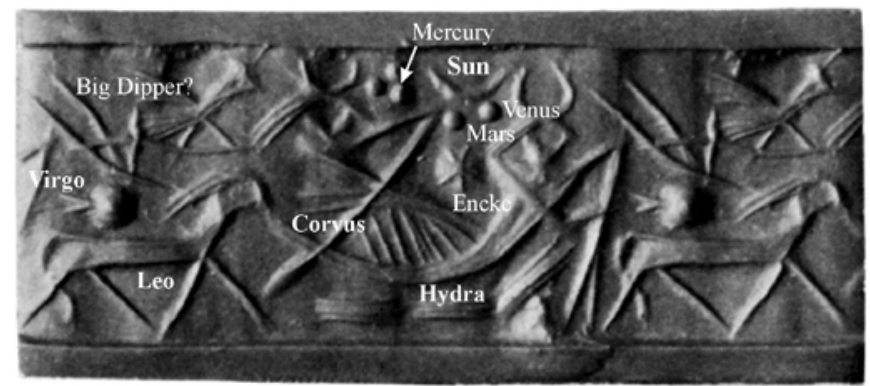

Figure 5. Mirror image of Frankfort No. 331 from Khafaje, Diyala region. Style: ED IIIa, 2600-2500 BC. "Horned long-haired god in boat holds steering oar; prow ends in human-shaped horned figure with punting pole; bird, plow, quadruped, and vase (drill-hole) outside boat; crescent, star, and five drill-holes in field (see pp. 8, 31, 33, 36). Lapis lazuli; $2.2 \times 1.1 \mathrm{~cm}$." Description by Frankfort [2]. 


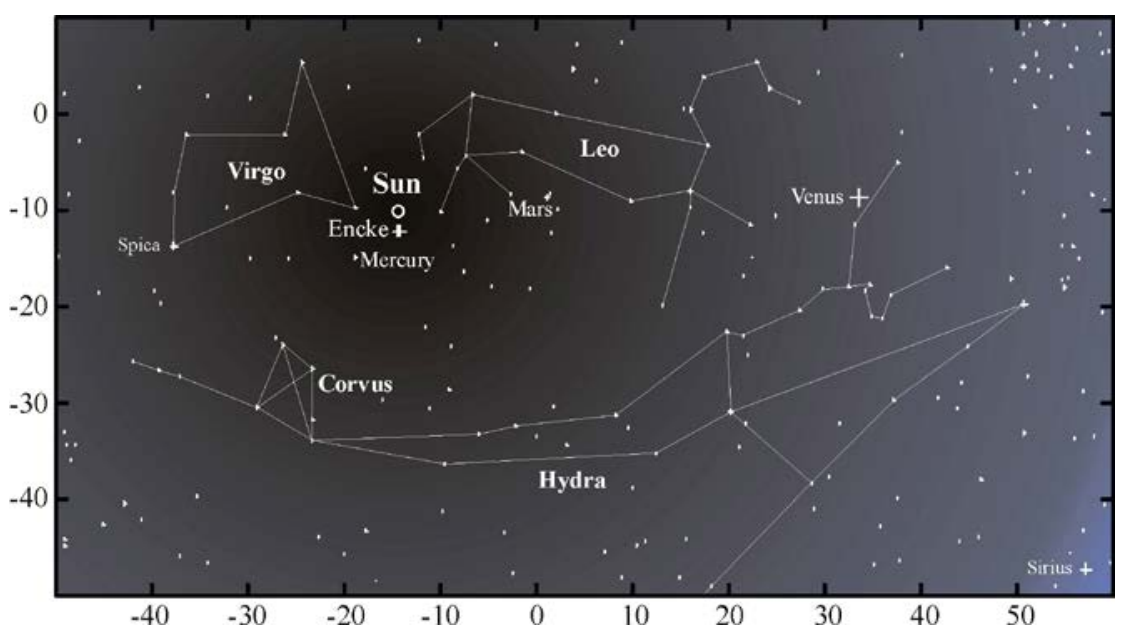

Figure 6. The total solar eclipse on 18 July, in 2654 BC, at 11.07, local mean solar time in Khafaje, Diyala region. Comet Encke, visible as a star just below the eclipsed sun, was that day moving along the line of sight towards the sun and the Earth. The tail was assumed to be 0.3 Astronomical Units long in the calculations, but no tail is visible, because it was hidden behind the head of the comet, with magnitude -1.6. To get Encke in this position just below the sun it was enough with a 10.0 days shift along the standard orbit, Henriksson [4]. (Zenith projection with stellar magnitudes $<4.5$.)

\section{Total Solar Eclipse in 2929 BC at the Gate of Heavens}

On the cylinder seals there are several examples of a gate with wings identified as the Gate of Heavens. Fortunately, there was a total solar eclipse depicted on one of these cylinder seals. It has No. 648 in von der Osten's catalogue, Figure 7 and 8.

On pages 114-115 von der Osten [1] writes: "The double doors of heaven, as shown on Sumerian seals of other collections, are well known. A winged door appears on the back of a recumbent bull before a seated deity on No. 648. A rope which seems to be attached to the winged door is held on one side by a seated deity and on the other by a minor deity."

The total solar eclipse in 2929 BC fits very well with the date Archaic or Sumerian. An identification of the Gate of Heavens with the constellation Gemini is obvious and its standing on a recumbent bull fits with the position of Gemini above the constellation Taurus (Bull).

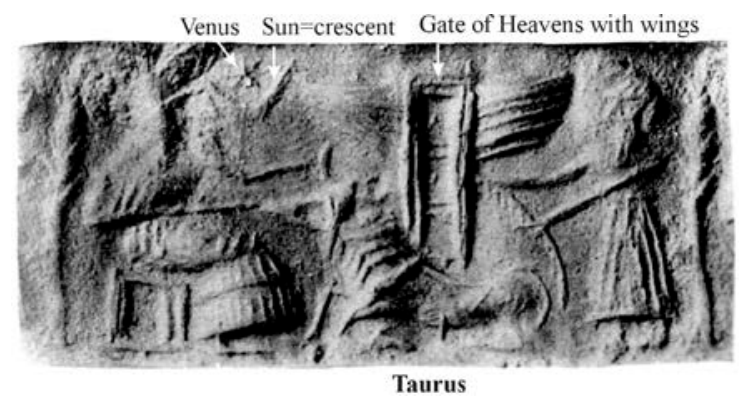

Figure 7. "A seated deity, wearing what seems to be a dress made of goat- or sheepskin, holds a rope attached to a winged door standing on a recumbent bull which faces the deity. A standing bearded figure in a long pleated robe holds a rope extending from the other side of the winged door. Before the deity appear a crescent and an eightpointed star. At the end of the scene is a tree." Seal No. 648 in von der Osten [1]). Limestone. 29 x 18 mm. 


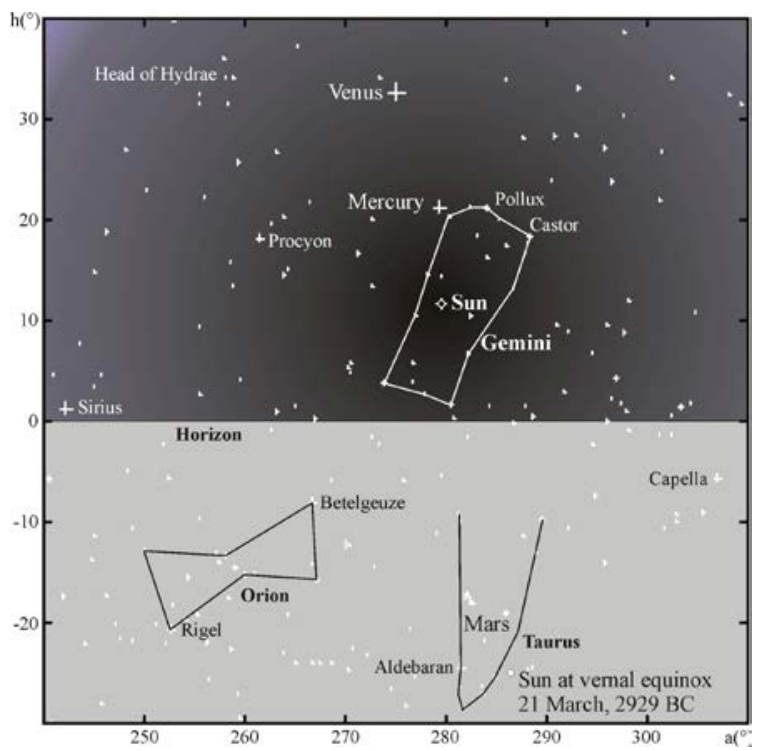

Figure 8. The total solar eclipse on 29 April, 2929 BC, at 17.32 local mean solar time in Eshnunna. The eclipse took place in Gemini, the Gate of Heavens.

\section{Total Solar Eclipse at the Pleiades in 2471 BC}

Frankfort [2] commented on page 30 the seal No. 352 from Khafaje, in the following way: "An eagle or the lion-headed eagle Imdugud is often shown between two ruminants, which it usually holds with its claws ... a combination of symbols referring to the god of fertility which is even now spoken of as a 'coatof-arms,' although its universal occurrence and great variety should long since have discredited an interpretation based on the knowledge of forty years ago."

The seal is Early Dynastic III (ED III), 2600-2350 BC, Figure 9.

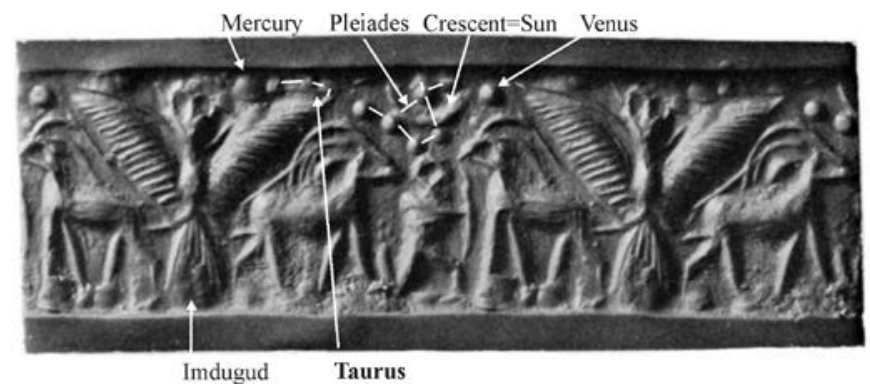

Figure 9. Frankfort No. 352 from Khafaje, Diyala region. "Lion-headed eagle holding a goat in either claw; kneeling figure holding indistinct object; crescent and seven drill-holes in field. Limestone; 2.5 x 1.4 cm." Frankfort [2]. Style: ED III, 2600-2350 BC. Identifications by the author.

Frankfort and von der Osten always identified a close group of seven drill-holes as the Pleiades. There exist at least 6 more drill-holes on seal No. 352, but according to von der Osten it is unusual that they are made just to fill empty space. In my opinion the artist has used the limited space in the upper part of the surface to depict from the left, Mercury, the horns of Taurus, the Pleiades, the crescent that represents the last visible phase before totality of the solar eclipse, and to the right, Venus, Figure 10. The main figure was Imdugud with the two goats and the kneeling man. 


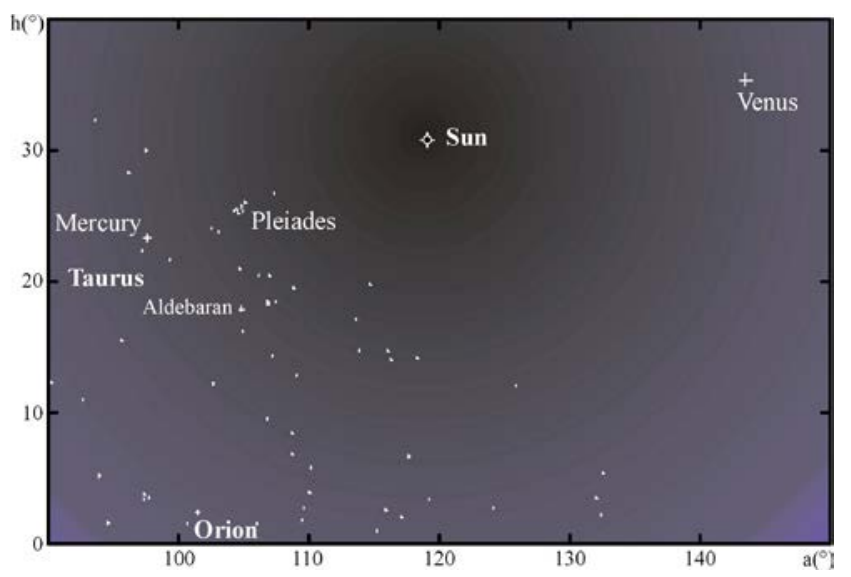

Figure 10. The eastern sky during the total solar eclipse on 10 March, in $2471 \mathrm{BC}$, at 08.55 local mean solar time in Khafaje, Diyala region. (Stellar magnitudes < 4.5.)

This solar eclipse was also total in Babylon and I have earlier identified this eclipse in the collection of omen texts Enuma Anu Enlil and expected to find it depicted on some of the Sumerian cylinder seals.

The total solar eclipse in Babylon in $2471 \mathrm{BC}$ at the Pleiades was the earliest of my identified total solar eclipses on the graph with $\Delta \mathrm{T}$, Henriksson [6] and [7].

In Enuma Anu Enlil, different kind of ominous risings and settings of the sun are mentioned, for instance in relation to clouds, but also that the sun was surrounded by 'the Stars' (Pleiades). W. van Soldt [8] has translated all the Tablets that mention solar omina and on Tablet 26(27) page 70 we can read: "[If the sun rises and ...] is surrounded by the Stars: there will be misfortune in that country."

The only possibility to see stars close to the sun is during a total solar eclipse. But a total solar eclipse visible at a specific site on the earth is a very rare event and a total solar eclipse in the morning close to a specific group of stars in the sky is an extremely rare event. In fact there was only one total solar eclipse close to the Pleiades, visible in ancient Babylon, and it took place on 10 March, Gregorian calendar, in 2471 BC, about two hours after sunrise, Figure 10.

\section{New Calibration of the Lunar Secular Acceleration in Longitude}

The lunar secular acceleration, calculated by different researchers, was earlier determined only from ancient solar and lunar eclipses. The most accurate of these calibrations was made by Carl Schoch in 1926 [9]. He calibrated his formulas from a conjunction of Spica and the moon in 283 BC, observed by Timocharis in Alexandria, and from an analysis of total solar eclipses back to $600 \mathrm{BC}$. He also used one partial solar eclipse in 1335 BC, mentioned in the annals of the Hittite king Mursilis I.

However, in 1975 Morrison and Ward [10] preferred to use transits of Mercury, 1677-1973, because they worked with Ephemeris Time (ET), with constant time flow, instead of the traditionally used Universal Time (UT), depending of the Earth's rotation rate. The advantage with UT is that it corresponds directly to the observed time given in the ancient sources, while ET needs to be calibrated. The great problem with this calibration is that it is impossible to avoid circular arguments for the ancient observations, and the period of telescopic observations used by Morrison and Ward in combination with mechanical pendulum clocks, is only about 300 years and this time interval is so short that non-tidal forces have disturbed the result in a significant way. The most important non-tidal effect on the Earth's rotation rate is the Earth's mean temperature and it has unfortunately been increasing since the Little Ice Age, with its coldest period around 1680, to the modern time with global warming. That means a maximum non-tidal disturbance in the Earth's rotation rate during the interval that Morrison and Ward used for their calibration.

The present author has developed a computer program for calculation of ancient solar eclipses using the theory of Schoch [9], but with modern parameters included and with a much more complete correction for the perturbations in the lunar orbit with about 450 terms. 
This program was finished in June 1985 and it has since been successfully tested against well-defined modern and ancient observations of total solar eclipses back to 3653 BC [3], [4], [11], [12], [13], [6], [7], [14] and [15]. Schoch's formulas are calibrated with UT as the time standard. He used the lunar apparent secular acceleration in his calculation of the lunar longitude. Schoch showed with one example how his lunar apparent secular acceleration, $24.40 / \mathrm{cy}^{2}$, and his value for the solar secular acceleration, 2.98 " $/ \mathrm{cy}^{2}$, could be used to calculate the lunar sidereal secular acceleration, $-29.68 \mathrm{l} / \mathrm{cy}^{2}$.

However, Schoch had a third-order term in his formula for the lunar longitude, introduced by Simon Newcomb as a fitting parameter. This means that the lunar sidereal secular acceleration is time dependent in Schoch's theory and the value $-29.68 " / \mathrm{cy}^{2}$ is only exactly valid around the year 1800.0 . The main purpose for Schoch was to get the best set of formulas for calculations of ancient solar eclipses. He was probably less interested in the real physical acceleration of the Moon.

In July 2011 the present author decided to try to find the true physical constant value for the sidereal secular acceleration of the Moon without the third-order term. The principle was to successively diminish the third-order term, without losing totality for 11 important and well-defined total solar eclipses and to calculate a new value for the constant secular acceleration term. Fortunately, it was possible to get totality for all these solar eclipses without the third-order term. A new calculation was made with 27 important total solar eclipses in order to find an improved value for the lunar secular acceleration that gave the highest combined magnitude of the eclipses.

Finally, a set of 33 sensitive total or almost total solar eclipses was selected between AD 878 and 3653 $\mathrm{BC}$, and a new search was started to find the value of the lunar secular acceleration, without a thirdorder term, that resulted in the maximum combined magnitude.

It was the apparent lunar secular acceleration that was optimized in these calculations and it was necessary to calculate almost one thousand solar eclipses to reach the final result.

The new value for the lunar apparent secular acceleration became 23.955 "/ $\mathrm{cy}^{2}$, which corresponds to 30.128 " cy $^{2}$ for the lunar sidereal secular acceleration.

However, if this value should be used as a physical parameter it is necessary to determine its statistical errors.

In 2014 the absolute errors were determined and in 2016 the mean errors were calculated, see Figure 11 .

The new value for the lunar sidereal secular acceleration, $-30.128 \pm 0.0035$ "/ $\mathrm{cy}^{2}$, gives an even better agreement with the ancient observations than with Schoch's original formula. The new formula for the lunar longitude is valid at least during the last 5650 years with a timing error of less than two minutes. Some of the identified ancient solar eclipses can be seen in Figure 12.

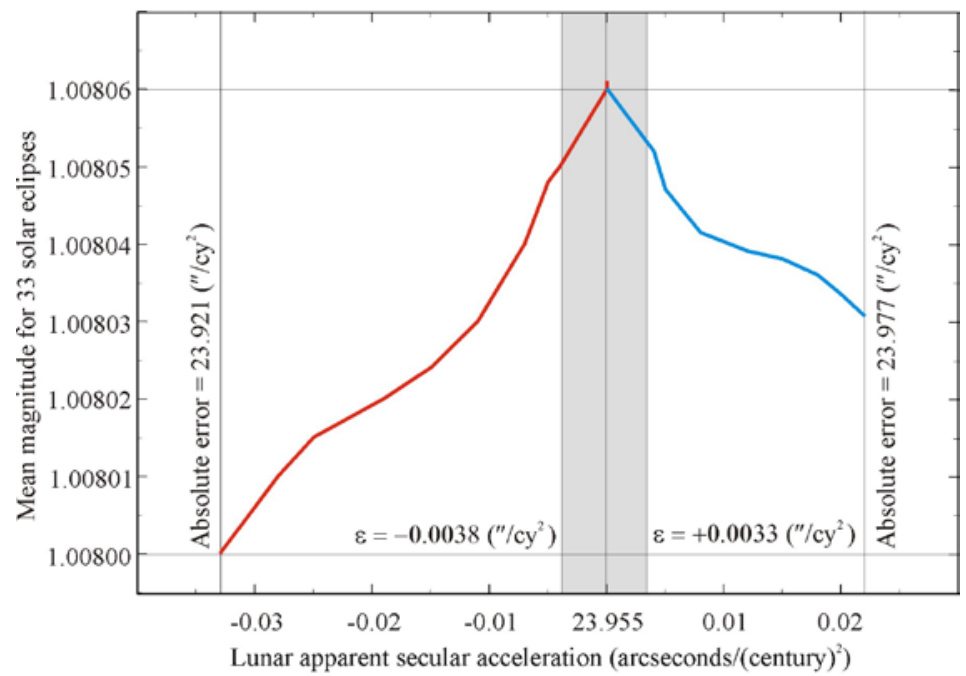

Figure 11. The distribution of the mean magnitudes for the 33 total or almost total solar eclipses, 878 AD - 3653 $\mathrm{BC}$, as function of the lunar apparent secular acceleration in longitude, are not symmetrical around the maximum value, $23.955 " / \mathrm{cy}^{2}$. 


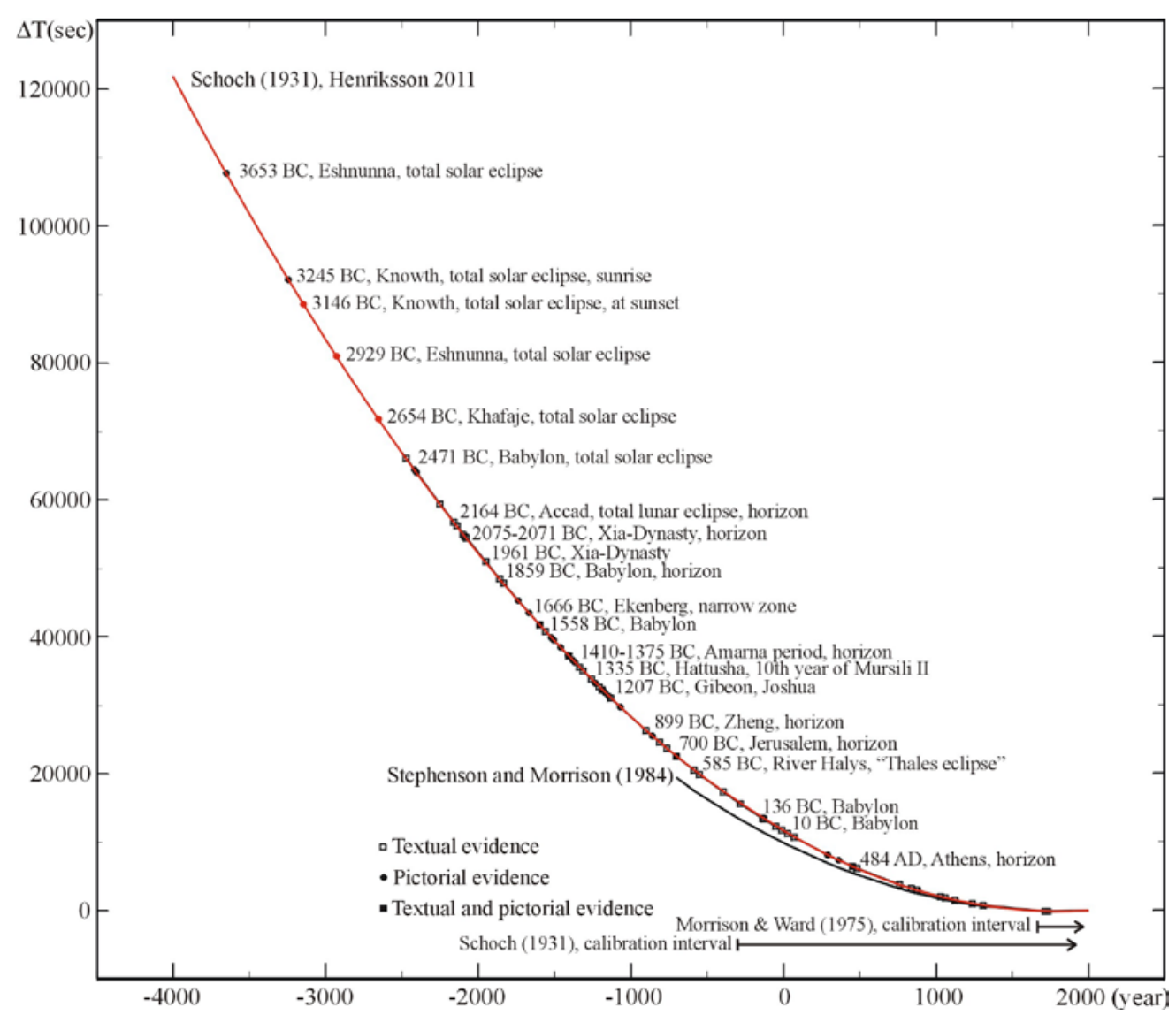

Figure 12. The parabolic time-shift, $\Delta \mathrm{T}$, due to the deceleration of the rotation of the Earth as a function of time with the coefficient 36.28 from Schoch [9]) and 31.0 from Stephenson and Morrison [16]. The unit for the coefficients is seconds/(century) $)^{2}$ and the time is reckoned from 1800.0. The symbols correspond to identified solar eclipses, mostly total or annular. Most of the identified eclipses can be found in Henriksson [3], [4], [11], [12], [13], [6], [7], [14] and $[15]$.

The data points marked with a red dot have not been included in the original analysis and can be considered as independent confirmations of the validity of the new calibration.

\section{Tidal Acceleration of the Moon from Lunar Laser Range Measurements}

Among the scientific instruments onboard Apollo 11, which performed the first landing on the moon on 20 July 1969, there was a reflecting prism. There are now five reflecting prisms on the surface of the moon, the last arrived in 1973.

A modified version of the Third Law of Kepler has been used in the calculation of the Lunar Laser Range (LLR) distances. It is therefore necessary to have a good enough value for the lunar secular acceleration in longitude.

An American and a French research team have performed parallel investigations. In the weighted mean I give the value, $-25.858 \pm 0.003 / \mathrm{cy}^{2}$, by Chapront et al. [17] weight three because its numerical value should be three times more accurate than -25.85 " $/ \mathrm{cy}^{2}$ by Williams et al. [18] as this value has only two decimals and with no error estimate.

The weighted mean of the lunar secular acceleration from the LLR-measurements in the Earth-Moon inertial system is: $-25.856 \pm 0.003 " / \mathrm{cy}^{2}$

\section{Relativistic Effects in the Earth-Moon System According to Nordtvedt}


In my earlier papers Henriksson [6] [7] [19] I have used the precession of the geodesic in the Earth-Moon inertial system caused by the Sun, according to Einstein [20] and de Sitter [21], -1.92 "/cy ${ }^{2}$, published by Nordtvedt [22]. In another paper by Nordtvedt [23] he presented a complete formula, to four decimal precision, for the relativistic effects in the Earth-Moon-system including also the Solar tidal acceleration and the precession of the geodesic caused by the mass of the Earth. However, Nordtvedt did not publish any numerical results from these complete formulas. I have now calculated all these relativistic effects with four decimal precision to avoid rounding effects.

The three components are: $-1.9189,+0.1177$ and $-0.0006 " / \mathrm{cy}^{2}$. The total relativistic effect on the lunar secular acceleration in longitude is $2 \mathrm{x}-1.8018 \mathrm{"} / \mathrm{cy}^{2}=-3.6036 " / \mathrm{cy}^{2}$.

\section{Einstein's Field Equations and Fierz and Pauli's Spin 2 Particles}

The original relativistic formulas by Einstein [20] were derived for a static Universe with a cosmological constant that exactly balanced the attraction of all gravitational forces. In this theory the mass of the graviton was absent. The investigation by Dirac [24] on relativistic wave equations for particles with arbitrary spin was followed up by Fierz [25]. Fierz and Pauli [26] studied particles with spin 2 and $3 / 2$. They investigated in detail the special case with a spin 2 particle with rest-mass zero under conditions identical with Einstein's first approximation of the gravitational equations. The equations for the gravitational field deviate from a Euclidean metric in small quantities of the first order Einstein [20]. Fierz and Pauli write in their summary. " ... In the particular case of spin 2, rest-mass zero, the equations agree in the force-free case with Einstein's equations for gravitational waves in General Relativity in first approximation; the corresponding group of transformations arises from infinitesimal co-ordinate transformations."

In a paper by H. van Dam and M. Veltman [27] they presented a study of massive and mass-less Yang-Mill's and gravitational fields. In the introduction they write concerning the case of the gravitational field. "A zero-mass theory has particles of spin 2 with two different states of polarization while finite-mass spin 2 particles have five polarization states." After the calculations in section 3 they conclude: "Thus in the massive case (but with extremely small mass) the bending of a ray of light passing near the sun is $3 / 4$ of that predicted in the mass-less case." ... "It turns out that also here the limit of zero-mass is discretely different from the zero-mass case, .... This implies that the bending of light rays near the sun and the perihelion movements of Mercury is distinctly different for zero-mass gravitons as compared to infinitesimal small mass gravitons. Experiment tells us then what theory to take, and the result is of course the zero-mass theory. We may conclude that the graviton has rigorously zero-mass."

After these investigations by great authorities, including Nobel laureates, and a convincing negative confrontation between theory and reality in the solar system, it is completely natural that the mass of the graviton was considered to be exactly zero in later theories in cosmology.

However, two years later, a scientist from the Institute of Nuclear Physics in Novosibirsk, A. I. Vainshtein [28], published a paper, with only $1 \frac{1}{2}$ pages, in which he solved the problem with the discontinuity between the theory for zero-mass graviton and the theory with a small but non-zero mass graviton. He realized that the reason for the discontinuity was that van Dam and Veltman had performed their calculations in the linear approximation. He writes: "We see that the perturbation theory does not suit for the consideration of zero-mass limit because higher orders contributions are singular in the graviton mass."

He concludes: "It may be shown that the higher orders of perturbation theory give a series in parameter $\rho_{\mathrm{g}} / \mathrm{m}^{4} \rho^{5}$ where $\rho_{\mathrm{g}}=k M / 4 \Pi$ is the Schwartschild radius. Therefore the perturbation theory does not work in the zero-mass limit."

The results presented in Vainshtein's paper became very important after the discovery by Riess et al. [29] and Perlmutter et al. [30] that the universe is accelerating. (Nobel prize 2011.)

Different theories with a non-zero mass graviton offered alternatives to the most accepted hypothesis called "Dark Energy" as the explanation for the acceleration. The discovery of a possible existence of non-zero mass gravitons is now called the "Vainshtein effect".

\section{Modified Theory of Gravity}


According to Dvali et al. [31], "Recent observations suggest that the Universe is accelerating on the scales of the present cosmological horizon [1]. This indicates that, either there is a small vacuum energy (or some 'effective' vacuum energy), or that conventional laws of Einstein gravity get modified at very large distances, imitating a small cosmological constant $[2,7,8]$. The first possibility is unnatural in the view of quantum field theory ${ }^{1}$, since the required value of vacuum energy $\sim 10^{-12} \mathrm{GeV}^{4}$ is unstable under quantum corrections. This unnaturalness goes under the name of the Cosmological Constant Problem.

In this respect the second approach, of modifying gravity in far infrared can be more promising since it is perturbatively stable under quantum corrections. The unnaturally small value of the vacuum energy is replaced by the idea that laws of conventional gravity break down at very large distances; beyond a certain crossover scale $r_{c}$. The value of $r_{c}$ is perturbatively-stable i.e. it does not suffer from cut-off sensitive corrections experienced by vacuum energy."

Dvali et al. [31] write in the abstract: "Cosmologically motivated theories that explain small acceleration rates of the Universe via modification of gravity at very large, horizon or super horizon, distances can be tested by precision gravitational measurements at much shorter scales, such as the Earth-Moon distance. Contrary to the naive expectation the predicted corrections to the Einstein metric near gravitating sources are so significant that they might fall within the sensitivity of the proposed Lunar Ranging experiments. The key reason for such corrections is the van Dam-Veltman-Zakharov discontinuity present in linearized versions of all such theories, and its subsequent absence at non-linear level ala Vainshtein."

For a radius of cross-over, or horizon, of $6 \mathrm{Gpc}\left(1.85 \times 10^{28} \mathrm{~cm}\right)$ the expected extra cosmological precession of the Moon is $-0.52 " / \mathrm{cy}^{2}$ according to formulas (13), (14) and (15) in Dvali et al. [31]. This corresponds to an additional lunar radial acceleration of $+0.76 \mathrm{~mm} / \mathrm{yr}$, Henriksson [6] [7].

\section{Radius of Crossover and the Mass of the Graviton}

If the LLR measurement is corrected for the relativistic effect, -3.6036 "/ $\mathrm{cy}^{2}$, we get the lunar secular acceleration, $-29.6938 \pm 0.003 " / \mathrm{cy}^{2}$. If this value is subtracted from the observed lunar sidereal secular acceleration $-30.128 \pm 0.0035 \mathrm{"} / \mathrm{cy}^{2}$, calibrated by Henriksson in 2011 , we get $-0.6685 / \mathrm{cy}^{2}$ with the total uncertainty $\pm 0.0046 / \mathrm{cy}^{2}$. Dvali et al. [31] predicted in their theory for modified gravity an additional cosmological precession of the Earth-Moon-system. I have now calculated this effect as -0.5227 " $/ \mathrm{cy}^{2}$, from their formulas in chapter 3 , with the radius of crossover, $r_{\mathrm{c}}=6 \mathrm{Gpc}\left(1.85 \times 10^{28} \mathrm{~cm}\right)$.

If the observed additional acceleration, $-0.6685 \pm 0.0046 " / \mathrm{cy}^{2}$, is interpreted as the cosmological precession, the corresponding radius of crossover is $4.69 \mathrm{Gpc}$ or 15.3 billion light years. If we accept that the Age of the Universe is 13.8 billion years and that the radius of the Universe is 46.5 billion light years we can conclude that the acceleration of the Universe started 4.54 billion years after the Big Bang.

According to chapter 4 in Dvali et al. [31], the mass of the graviton $\mathrm{m}_{\mathrm{g}}=1 / \mathrm{r}_{\mathrm{c}}$.

After converting units it is found that the Mass of the Graviton is $1.306 \pm 0.009 \times 10^{-56}$ grams.

\section{Conclusions}

Some of the motifs on the Sumerian cylinder seals can be identified as depictions of the sky during total solar eclipses and can be used to calibrate the lunar secular acceleration in longitude over a 5650 year long time interval. This value can be combined with the LLR measurements of the radial acceleration of the Moon, corrected for General Relativity, to test the Third Law of Kepler. The deviation corresponds to the cosmological acceleration of the Moon suggested by Dvali et al. [31] in a theory of Massive Gravity.

The massive graviton is 5-dimensional and needs a 5D space, but within distances $<r_{\mathrm{c}}$, a $4 \mathrm{D}$ theory is sufficient. For distances $>r_{\mathrm{c}}$, the gravitons begin to leak to the 5 th dimension and the gravitational forces between the galaxies are weakened resulting in the observed acceleration of the Universe.

The sign of the cosmological acceleration is negative which means that we find ourselves in the standard cosmological branch according to Lue and Starkman [32].

Acknowledgements. I am very grateful to Dr Johan Flemberg, Department of Classical Archaeology and Ancient History at Uppsala University, for his help in 1986 to find the reference to the protoliterate 
cylinder seal and to associate professor Mary Blomberg, at the same department, for her improvements of my English. I am also grateful to my colleague Dr Andreas Korn, Department of Physics and Astronomy, Uppsala University, for his critical reading of the sections 10, 11 and 14.

\section{References}

1. H. von der Osten, Ancient Oriental Seals in the Collection of Mr. Edward T. Newell. The University of Chicago Oriental Institute Publications Vol. XXII, 1934.

2. H. Frankfort, Stratified Cylinder Seals from the Diyala Region, The University of Chicago Oriental Institute Publications, Volume LXXII, 1955.

3. G. Henriksson, "Prehistoric constellations on Swedish Rock-carvings,"in Actes de la V'me conférence de la SEAC, Gdańsk, 5-8 septembre 1997 (Światowit supplement series H: Anthropology, 2), Warsaw, 1999, pp. 155-173.

4. G. Henriksson, "Solar eclipses, supernova and Encke's comet on Swedish rock Carvings," in Proceedings of the Fifth Oxford International Conference on Archaeo-astronomy, Santa Fe, August 1996, Carolina Academic Press, Durham, North Carolina, 2005, pp. 475-485.

5. A. Jeremias, "Handbuch der Altorientalischen Geisteskultur," J. C. Hinrichsch Buchhandlung, Leipzig, 1913, p. 247.

6. G. Henriksson, "A new test of Einstein's theory of relativity by ancient solar eclipses," in Cosmology across Cultures, Astronomical Society of the Pacific Conference Series vol. 409, 2009, pp. 166-171.

7. G. Henriksson, "Einstein's Theory of Relativity confirmed by Ancient Solar Eclipses," in Journal of Cosmology, 2010, Vol. 9, pp. 2259-2270, 2010.

8. W. H. van Soldt, "Solar Omens of Enuma Anu Enlil: Tablets 23 (24) - 29 (30)," Istanbul, 1995.

9. C. Schoch, "Die säkulare Accelaration des Mondes und der Sonne," Astronomische Abhandlungen, Ergänzungshefte zu den Astronomischen Nachrichten, Band 8, B2. Kiel, 1931.

10. L. V. Morrison and C. G. Ward,. "An analysis of the transits of Mercury 1677-1973," Mon. Not. R. Astr. Soc. 173, 1975, pp. 183-206.

11. G. Henriksson, "A new Chronology of the Old Babylonian Kingdom and Ur I-III based on identification of solar and lunar eclipses," in Proceedings of the SEAC 2002 Conference in Tartu. Tartu 2006.

12. G. Henriksson, "Chronology for the Egyptian Pharaohs of the Amarna period and the Israeli leaders Moses and Joshua by correlation with eight solar eclipses," in Proceedings of the SEAC 2004 Conference in Kecskemet. BAR International Series 1647, 2007, pp. 133-148.

13. G. Henriksson, "A new attempt to date the Xia, Shang and Zhou dynasties by solar eclipses," in Proceedings of the Oxford 8 and SEAC 2007 Conference in Klaipeda, Astronomy and Cosmology in Folk Traditions and Cultural Heritage, Archaeologica Baltica 10, pp. 105-109. Klaipeda, 2008.

14. G. Henriksson, "A Solar Eclipse in 700 BC Observed in Jerusalem and Southern Sweden," in Ancient Cosmologies and Modern Prophets, Proceedings of the 20th Conference of the European Society for Astronomy in Culture 2012, Ljubljana, 2013.

15. G. Henriksson, "Thales of Miletus, Archimedes and the Solar Eclipses on the Antikythera Mechanism," in Journal of Science and Engineering 4 (2014), pp 757-769. doi: 10.17265/2159-581X/2014. 12. 005

16. F. R. Stephenson, and L. V. Morrison, "Long-term changes in the rotation of the Earth: 700 B.C. to A.D. 1980," Phil. Trans. R. Soc. A 313, London 1984, pp. 47-70.

17. J. Chapront, M. Chapront-Touzé and G. Francou, "A new determination of lunar orbital parameters, precession constant and tidal acceleration from LLR measurements," Astron. Astrophys. 387, 2002, pp. 700-709.

18. J. G. Williams, D. H. Boggs and W. M. Folkner, "DE421 Lunar orbit, Physical Librations, and Surface Coordinates," IOM 335-JW,DB,WF-20080314-001, March 14, 2008.

19. G. Henriksson, "Lunar secular acceleration supports a modified theory for gravity," in Proceedings of the $49^{\text {th }}$ Rencontres de Moriond, La Thuile, March 22-29, 2014.

20. A. Einstein, "Die Grundlage der allgemeinen Relativitätstheorie," Annalen der Physik, 49, 1916.

21. W. de Sitter, Mon. Not. R. Astr. Soc. 77, 1916, p. 155.

22. K. Nordtvedt, "From Newton's Moon to Einstein's Moon,“ Physics Today, May 1996, Vol. 49:5, p. 29.

23. K. Nordtvedt, "The Relativistic Orbit Observables in Lunar Laser Ranging," ICARUS 114, 1995, 51-62.

24. P. Dirac, Proc. Roy. Soc. A, 155, 1936, p 447. 
25. M. Fierz, Helv. Phys. Acta, 12, 3, 1939.

26. M. Fierz and W. Pauli,. "On relativistic wave equation for particles of arbitrary spin in an electromagnetic field," Proc. R. Soc. Lond. A 1039 173, published 28 November 1939, pp. 211-232.

27. H. van Dam, and M. Veltman, "Massive and Mass-less Yang-Mills and Gravitational fields," Nucl. Phys. B22, 1970, pp. 397-411.

28. A. I.. Vainshtain, "To the problem of non-vanishing gravitation mass," Phys. Lett. 39 B, number 3, 1 May 1972, pp. 393-394.

29. A. G. Riess et al. (High-z Supernova Team), "Observational Evidence from Supernovae for an Accelerating Universe and Cosmological Constant," Astron. J. 116, 1009, 1998.

30. S. Perlmutter et al. (Supernova Cosmology Project), "Measurements of Omega and Lambda from 42 HighRedshift Supernovae," Astrophys. J. 517, 565, 1999.

31. G. Dvali, A. Gruzinov and M. Zaldarriaga, "The Accelerated Universe and the Moon," Phys. Rev. D, vol. 68, Issue 2, id. 024012, 2003.

32. A. Lue and G. Starkman, "Gravitational Leakage into Extra Dimensions: Probing Dark Energy Using Local Gravity," Phys. Rev. D 67, 064002, 2003. 